\title{
FOVO: A flexible real-time computer graphics rendering process
}

\author{
Robert Pepperell \\ Fovolab \\ Cardiff Metropolitan \\ University, UK \\ rpepperell@cardiffmet.ac.uk
}

\author{
Alistair Burleigh \\ Fovolab \\ Cardiff Metropolitan \\ University, UK \\ aburleigh@cardiffmet.ac.uk
}

\author{
Nicole Ruta \\ Fovolab \\ Cardiff Metropolitan \\ University, UK \\ niruta@cardiffmet.ac.uk
}

\author{
Tony Langford \\ Fovolab \\ Cardiff Metropolitan \\ University, UK \\ tlangford@cardiffmet.ac.uk
}

\section{INTRODUCTION}

Real-time rendering processes in computer graphics systems are often designed around the principles of linear projection (LP), that is, simulating linear light rays passing through 3D space and intersecting a two-dimensional surface in order to create an image.

For hundreds of years artists and technologists have also relied on the same principles to make images of the world. But LP does not necessarily represent 3D space as we experience it perceptually, and especially so in images with a wide field of view. 'Optically correct' image renderings limit both technical and artistical possibilities of expression of graphics designers, especially in applications like 3D image visualization in gaming (Taylor 2002). Users are often dissatisfied with the standard field of view used in computer games: if too narrow they miss a lot of the action and feel 'boxed in'; if too wide the centre of the image shrinks and the edges of the scene become stretched (Ljung 2017).

FOVO (Field of View Opened) takes a different approach to rendering. Rather than only modelling the behaviour of light, as in a conventional LP based process, it is designed to instead emulate visual experience more naturalistically by additionally simulating key characteristics of human visual perception in the output images at a deeper cognitive level. At the core of FOVO is a novel set of mathematical transformations that convert $3 \mathrm{D}$ space into a $2 \mathrm{D}$ format, in a way that provides far more visual realism, flexibility, and usability than a standard linear render.

Rendering with FOVO can overcome many of the problems associated with imaging technologies based only on LP, and can provide significant benefits. FOVO is based on scientific research that integrates the psychophysical study of visual perception, artistic knowledge, novel computer graphics techniques and user testing (Baldwin et al. 2016; Burleigh et al. 2018; Langford et al. 2018).
FOVO can be used to render a scene with the following benefits, to:

1. Create naturally wide fields of view in a variety of projections with an enhanced sense of embodiment.

2. Create a greater sense of depth, and more immersive and realistic projections of 3D space than is possible with standard linear projections.

3. Calibrate image geometry to accommodate different screen sizes and display formats.

4. Manipulate image geometry in new ways in order to make the best use of available screen space.

5. Provide a novel projective image making framework within which an infinite range of new image projections, including all standard mathematical projections.

6. Create wide angle curvilinear projections with no image resolution degradation.

7. Greatly improve distance judgements in the 3D space (offering more accuracy, with less fatigue).

We have collected substantial evidence to show a preference for FOVO compared to standard LP renderings.

\section{IMPROVED DISTANCE PERCEPTION}

In one study we hypothesised that FOVO images would provide an improved perception of distance compared to LP images. To test this we asked participants $(\mathrm{N}=40)$ to estimate perceived distance of a target object in a gaming virtual environment at $100^{\circ}, 120^{\circ}$ and $140^{\circ}$ horizontal field of view for both FOVO and LP. 
Our results showed that participants were 1.53 times more likely to get the correct answer and 1.69 times more likely to be accurate when looking at the FOVO images compared with the LP images. We also showed that the likelihood of getting the correct answer at wider fields of views $\left(140^{\circ}\right)$ vastly increased for FOVO images, while decreased for LP. We also found a learning effect, meaning that participants became more accurate at estimating target distance if looking at FOVO, but not at LP, where participants' accuracy performance vastly decreased. We showed that, on average, users slightly underestimated target distance when looking at FOVO images but largely overestimated it when looking at LP images, particularly at high field of views.

Overall, we found that FOVO images have the significant benefit of improved distance judgments compared to standard LP.

\section{NATURALNESS OF IMAGE GEOMETRY}

In a further study we hypothesised that people would prefer the wide field of view projections generated by FOVO. To test this, we conducted a series of experiments to investigate perceived sense of presence and naturalness associated with FOVO images.

In a large-scale online study $(\mathrm{N}=74)$, we found that people preferred FOVO images compared to standard linear perspective and fisheye projections. These results were even stronger when people had the possibility to compare the image with the experience of being in front of the real scene depicted in the image $(\mathrm{N}=26)$. FOVO images were rated as more comfortable to look at and with higher sense of presence than standard linear perspective and fisheye images.

When given the possibility to directly manipulate the geometry of the image to match their perception, $93.62 \%$ of our sample population $(\mathrm{N}=47)$ chose settings closer to FOVO settings. We found the same pattern of results when people manipulated the image geometry of the rendering of a virtual environment, with $73.86 \%$ of participants preferring FOVO as a more natural representation of the virtual space. The preferred average horizontal field of view for FOVO corresponded to $142^{\circ}$ of visual angle, compared to $115^{\circ}$ for linear perspective images.

Finally, in another study ( $\mathrm{N}=26)$ FOVO images were significantly preferred and judged to be more comfortable to look at compared to other standard image renderings like LP and fisheye projections. FOVO was rated as equally immersive as looking at an image presented on a cylindrical rear- projected screen, covering $180^{\circ}$ of participants' visual angle $(\mathrm{N}=8)$.

\section{CONCLUSION}

We have shown that FOVO images significantly improved distance judgements and are perceived as more immersive and realistic when presented on normal sized screens and compared to standard LP projections.

As a computer graphics process there are numerous potential applications for FOVO in any industry that uses computers to create or manipulate images, for example advertising, architecture, design, entertainment, communications, medicine and wherever there is a benefit in more accurately representing human visual experience.

When applied within a gaming environment, FOVO images represent natural vision more accurately and effectively and are preferred to conventional $\mathrm{LP}$ renderings.

It is important to note that improved distance perception, immersion and comfort were measured without any specialist equipment, headsets or glasses, and this provides numerous possibilities in cinematography and broadcasting for improved viewer experience. In medical imaging, the increased accuracy of FOVO images also raises the prospect of improved detection of cancers through endoscopy / laparoscopy, as well as more immersive training applications.

\section{REFERENCES}

Baldwin, J., Burleigh, A., Pepperell, R. and Ruta, N. (2016) The perceived size and shape of objects in peripheral vision. I-Perception, 7(4).

Burleigh, A., Pepperell, R. and Ruta, N. (2018) Natural Perspective: Mapping Visual Space with Art and Science. Vision, 2(2), 21.

Langford, T., Burleigh, A., Ruta, N. and Pepperell, R. (2018) New immersive media to broaden attention and awareness. In Proceedings of the Conference on Electronic Visualisation and the Arts, pp.323-330.

Ljung, K. (2017) Effects of Field-of-View in FirstPerson Video Games: A Study on Camera Field-ofView in Relation to Game Design. Undergraduate Uppsala.

Taylor, L. (2002) Video Games: Perspective, Pointof-view, and Immersion [e-Book]. University of Florida, Florida. 